\title{
Balthasar Baro, études réunies et présentées par B.
} LOUVAT-MOLOZET, P. PASQUIER

\section{Laura Rescia}

\section{(2) OpenEdition \\ Journals}

\section{Edizione digitale}

URL: https://journals.openedition.org/studifrancesi/22566

DOI: 10.4000/studifrancesi.22566

ISSN: 2421-5856

\section{Editore}

Rosenberg \& Sellier

\section{Edizione cartacea}

Data di pubblicazione: 1 avril 2020

Paginazione: 168-169

ISSN: 0039-2944

\section{Notizia bibliografica digitale}

Laura Rescia, «Balthasar Baro, études réunies et présentées par B. LOUVAT-MOLOZET, P. PASQUIER», Studi Francesi [Online], 190 (LXIV | I) | 2020, online dal 01 avril 2020, consultato il 03 août 2021. URL: http:// journals.openedition.org/studifrancesi/22566 ; DOI: https://doi.org/10.4000/studifrancesi.22566

Questo documento è stato generato automaticamente il 3 août 2021.

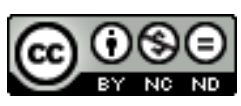

Studi Francesi è distribuita con Licenza Creative Commons Attribuzione - Non commerciale - Non opere derivate 4.0 Internazionale. 


\title{
Balthasar Baro, études réunies et présentées par B. LOUVAT-MOLOZET, P. PASQUIER
}

\author{
Laura Rescia
}

\section{NOTIZIA}

Balthasar Baro, études réunies et présentées par B. LOUVAT-MOLOZET, P. PASQUIER, “La Licorne" 132, 2018, 208 pp.

1 La figura di Balthasar Baro viene in genere ricordata anche dagli specialisti in relazione al suo ruolo di segretario di Honoré d'Urfé, a cui spettò il ruolo di pubblicare postuma la quarta parte dell'Astrée, e che volle comporre la quinta e ultima parte di sua propria mano. Tuttavia, egli non fu un personaggio secondario nell'agone letterario della prima metà del Seicento, la sua opera essendo più ricca e composita di quel che si ritenga. Fu drammaturgo prolifico tra il 1628 e il 1650, e non rimase ancorato alla sua città natale, Toulouse, dove pure fu particolarmente attivo: tre delle sue pièces figurano nel repertorio dell'Hôtel de Bourgogne negli anni Trenta-Quaranta; accademico fin dal 1636, Baro svolse un ruolo di primo piano nelle vicissitudini dell'Horace di Corneille e partecipò alla querelle $d u$ Cid; ed infine, ci ricordano i curatori di questo volume nell'introduzione ricevette l'approvazione di un critico feroce come l'abbé d'Aubignac.

Questo numero tematico della rivista dell'Université de Rennes raccoglie i lavori di un convegno, svoltisi a Montpellier tempo fa ma solo ora pubblicati, che intendeva aggiornare le notizie e lo sguardo critico sull'opera romanzesca e drammaturgica e sulla sua carriera. Ricordiamo inoltre la recente pubblicazione del primo volume dell'edizione moderna del teatro di Baro, sotto la direzione della stessa Louvat-Molozet, apparso per le edizioni Classiques Garnier nel 2014.

A guisa di prologo, l'articolo di Pierre ESCUDE, Baro, Monluc, Godolin: le Cléosandre de 1624 et le libertinage toulousain, pp. 15-30 è dedicato alla collaborazione di Baro al libretto per un 
balletto messo in scena a Toulouse durante il carnevale del 1624. L'estetica del balletto è ambigua, a cavallo tra l'ideologia libertina tolosana e il richiamo ad una poetica mondana di ritorno all'ordine e all'ortodossia.

Gli articoli sono raggruppati in tre sezioni. La prima, dedicata a Baro continuatore e adattatore dell'Astrée, si apre con un articolo di Tomás Gonzalo SANTOS, La Conclusion de L'Astrée de Balthasar Baro et les attentes des lecteurs contemporains, pp. 33-41, che mostra puntualmente come egli si fece erede di tutti gli elementi tematici e stilistici dei primi quattro libri, per proporre una conclusione che riesce a fornire un epilogo alla quasi totalità degli intrecci urfeiani. Madeleine BERTAUD, Que devient la matière politique de L'Astrée dans la Suite de Baro?, pp. 43-52, si concentra su di un unico aspetto dell'eredità di d'Urfé, quello politico, per constatare come il suo segretario lo abbia sviluppato, prolungato e talvolta attualizzato, alla luce degli avvenimenti a lui contemporanei, rendendosi dunque fedele continuatore del dettato di d'Urfé. È invece dedicato alle poetiche romanzesche e drammaturgiche l'articolo di Chrystelle BARBILLON, Écriture narrative, écriture dramatique : passerelles, parallèles et passages dans les premières œuvres de Balthasar Baro, pp. 53-68, che si concentra in particolare sul passaggio in scena di episodi dell'Astrée nelle prime due pièces di Baro, la tragicommedia Célinde (1629) e la pastorale Clorise (1632).

5 Si apre quindi la seconda sezione del volume, dedicata alla scrittura drammaturgica. Bénédicte LouvaT-MolozaY, Les "poèmes dramatiques» de Baro: dénomination et pratiques génériques, pp. 71-84 considera come la denominazione di «poèmes dramatiques» voluta da Baro per quelle che sono oggi considerate tragicommedie, sia la più appropriata a dar conto della sua poetica eterogenea e tuttavia non completamente sovrapponibile a quella tragicomica dell'epoca. Si concentra invece sulla tragedia l'articolo successivo, in cui Dominique MonconD'HuY, Baro et la tragédie, pp. 85-93 a partire dalla constatazione del quasi completo rifiuto di tale genere da parte di Baro, ritiene tale posizione essere frutto del rifiuto delle norme e della volontà di affermare uno spazio personale di libertà. Hélène VISENTIN, La conception du spectacle dans le théâtre de Balthasar Baro, pp. 95-106, propone, attraverso un'analisi trasversale degli elementi spettacolari del suo teatro, di considerarlo un drammaturgo avanguardista, quale iniziatore del procedimento del teatro nel teatro e della presenza del meraviglioso cristiano. Jean GARAPON, Visages du féminisme héroïque dans le théâtre de Balthasar Baro, pp. 107-118, esamina le figure di «femmes fortes» presenti nell'insieme della sua scrittura drammaturgica, per constatare come le sue eroine evolvano da un profilo nettamente femminista verso un ideale femminile più discreto e riservato, dove coraggio e determinazione lasciano spazio alla solitudine e all'interiorità. L'intervento di Véronique MOUTOT-NARCISSE, Comment l'esprit vient aux pères: conflits d'autorité dans Célinde de Balthasar Baro, pp. 119-132 è focalizzato sul tema del conflitto con l'autorità paterna nella Célinde, dove la rivolta dei figli verrà armonizzata con il potere parentale grazie all'intervento di un'autorità pubblica esterna alla famiglia. Alla stessa pièce è dedicato l'articolo di Ana CONBOY, Le double hommage de Baro: pratique théâtrale et théâtre irrégulier dans la (méta)théâtralité de Célinde, pp. 133-145, che analizza il contributo apportato da Baro all'estetica dell'irregolarità, evocando, sebbene in assenza di documenti, l'ipotesi che questa pièce abbia potuto provocare una querelle.

6 La terza parte del volume è interamente dedicata all'unica tragédie sainte di Baro, Saint Eustache Martyr, che viene analizzata in relazione al problema delle rappresentazioni sacre sulla scena parigina alla fine degli anni Trenta. Pierre PASQUIER, Baro et la comédie 
de dévotion: de l'innovation à la tradition?, pp. 149-167 indica la strada percorsa da Baro rispetto ai suoi predecessori: con questa pièce egli rinuncia al tragico e alle regole classiche allora nascenti per adottare un'estetica a cavallo tra tradizione (quella del teatro medievale) e innovazione (come la mescolanza di toni galanti e mistici).

7 Claude Bourqui, Le Saint Eustache martyr de Baro et l'évolution de la "tragédie de dévotion»: dramaturgie religieuse contre dramaturgie profane, pp. 171-180, dimostra come, sia per quanto riguarda la dispositio che per l'inventio, la pièce sia risolutamente antiaristotelica; Anne TEULADE, Deux modulations pour une esthétique hybride: l'articulation du profane et du religieux dans Saint Eustache martyr de Balthasar Baro et La Mayor dicha en el monte de Lope de Vega, pp. 181-194, compara la pièce a un adattamento di Lope della vicenda del santo, per evidenziare due estetiche opposte, dove Baro cerca di attirare la devozione nella sfera del profano.

8 A conclusione, un contributo di Arlette JouAnNA, Balthasar Baro et la charge de trésorier de France, pp. 197-204, dedicato al ruolo a cui aspirava al termine della sua carriera letteraria. 